\title{
Qishen Huanwu capsule reduces pirarubicin-induced cardiotoxicity in rats by activating the PI3K/Akt/mTOR pathway
}

\author{
Feng Wang ${ }^{1}$, Lixin Wang ${ }^{1,2}$, Yue Jiao ${ }^{3}$, Zhirong Wang ${ }^{2}$ \\ ${ }^{1}$ Hebei University of Chinese Medicine, Shijiazhuang, China; ${ }^{2}$ Department of Cardiovascular Disease, Hebei Province Cangzhou Hospital of \\ Integrated Traditional Chinese and Western Medicine, Cangzhou, China; ${ }^{3}$ Experimental Research Center, China Academy of Chinese Medical \\ Sciences, Beijing, China \\ Contributions: (I) Conception and design: F Wang; (II) Administrative support: L Wang; (III) Provision of study materials or patients: Y Jiao; (IV) \\ Collection and assembly of data: F Wang, Z Wang; (V) Data analysis and interpretation: F Wang; (VI) Manuscript writing: All authors; (VII) Final \\ approval of manuscript: All authors. \\ Correspondence to: Lixin Wang. Department of Cardiovascular Disease, Hebei Province Cangzhou Hospital of Integrated Traditional Chinese and \\ Western Medicine, Cangzhou, China. Email: wlx006@163.com.
}

Background: One of the common adverse reactions to anthracyclines, a group of chemotherapeutics, is cardiotoxicity. Cancer patients receiving anthracycline-based chemotherapeutic regimens often discontinue treatment due to cardiotoxicity. How to prevent and reduce the cardiotoxicity of anthracyclines is one of the hot topics in the field of onco-cardiology. Traditional Chinese medicine can reduce the toxic side effects of chemotherapeutics. The present study aimed to investigate the protective effect of Qishen Huanwu capsule (QSHWC) on pirarubicin (THP)-induced myocardial injury in rats and the underlying mechanisms.

Methods: Forty-eight male Sprague-Dawley (SD) rats were randomly divided into six groups: control group, THP, low-dose QSHWC, moderate-dose QSHWC, high-dose QSHWC, and LY294002 [phosphatidylinositol 3-kinase (PI3K) inhibitor] (n=8 each). Echocardiographic examination was performed to examine heart structure and function. Hematoxylin and eosin (HE) staining was conducted to examine histopathological changes in myocardial tissue. Immunofluorescence staining was carried out to examine the expression of the autophagosome-specific marker protein microtubule-associated protein 1 light chain 3 (LC3). Western blot was performed to analyze the expression of LC3-I, LC3-II, PI3K, phosphorylated (p)PI3K, protein kinase B (Akt), p-Akt, mammalian target of rapamycin (mTOR), and p-mTOR.

Results: Compared with the control group, the THP group had a higher left ventricular end-systolic diameter (LVESD), lower left ventricular ejection fraction (LVEF), lower left ventricular fractional shortening (LVFS), and inferior heart function. In addition, compared with the control group, the THP group had significantly higher LC3 protein expression, a significantly higher LC3-II/LC3-I ratio $(\mathrm{P}<0.05)$, and significantly lower p-PI3K, p-Akt, and p-mTOR $(\mathrm{P}<0.05)$. QSHWC attenuated the THP-induced decline in heart function, downregulated LC3 protein in rat myocardial tissue, decreased the LC3-II/LC3-I ratio, and increased p-PI3K, p-Akt, and p-mTOR. In the LY294002 group, the above effects of QSHWC were reversed.

Conclusions: QSHWC alleviated THP-induced myocardial injury. The underlying mechanism was related to the activation of the PI3K/Akt/mTOR pathway and the mitigation of the excessive autophagy of cardiomyocytes caused by THP.

Keywords: Qishen Huanwu capsule (QSHWC); phosphatidylinositol 3-kinase/ protein kinase B/mammalian target of rapamycin signaling pathway (PI3K/Akt/mTOR signaling pathway); pirarubicin; cardiotoxicity; autophagy

Submitted Aug 07, 2020. Accepted for publication Sep 11, 2020.

doi: 10.21037/apm-20-1746

View this article at: http://dx.doi.org/10.21037/apm-20-1746 


\section{Introduction}

Pirarubicin is an anthracycline antibiotic, which plays an anti-tumor role by interfering with the synthesis of tumour cell DNA, and is widely used in the treatment of blood system malignant tumors and solid tumors. Unfortunately, the clinical effectiveness of THP is limited by its dose-related cardiotoxicity (1). Mechanisms by which THP induces cardiac side-effect have been investigated extensively, but a definitive picture has yet to emerge. Oxidative stress and apoptosis have been identified to be involved in THP-induced cardiotoxicity (2). Autophagy is a lysosomal-dependent protein degradation pathway, which plays an important role in doxorubicin-induced cardiomyopathy. Up-regulation of PI3K/AKT/mTOR pathway can inhibit excessive autophagy of cardiomyocytes caused by doxorubicin and reduce myocardial injury (3). However, the mechanism of autophagy in THP-induced cardiotoxicity has not been reported.

Qishen Huanwu capsule (QSHWC) (Ji Yao Zhi Zi Z20050798, batch number 030310) is an in-hospital preparation developed by Cangzhou Hospital of Integrated Traditional Chinese and Western Medicine in Hebei Province. QSHWC is derived from Buyang Huanwu decoction and has the effects of invigorating Qi and nourishing Yin as well as removing blood stasis and eliminating phlegm. It is mainly used to treat stroke and coronary heart disease due to Qi deficiency and blood stasis. Quercetin (4), kaempferol (5), and other components contained in QSHWC have the effect of reducing the cardiotoxicity of anthracyclines. In view of these findings, the present study intended to evaluate whether QSHWC could alleviate pirarubicin (THP)-induced myocardial injury in rats and explore the potential underlying mechanisms. We present the following article in accordance with the ARRIVE reporting checklist (available at http://dx.doi. org/10.21037/apm-20-1746).

\section{Methods}

Forty-eight specific-pathogen-free (SPF) male Sprague-Dawley (SD) rats, aging 6 weeks, weighing 160-180 g, were purchased from Beijing Vital River Laboratory Animal Technology Co., Ltd. (certificate number: SCXK (Beijing) 2016-0006).

\section{Ethics review}

The experimental rats received a standard rat diet and had free access to drinking water. The rats were kept in a pathogen- free room with a constant temperature of $25 \pm 3{ }^{\circ} \mathrm{C}$ and a light/dark cycle of $12 / 12 \mathrm{~h}$. All the procedures were carried out according to the Guide for the Care and Use of Laboratory Animals published by the U.S. National Institutes of Health and were reviewed and approved by the Ethics Committee of Cangzhou Hospital of Integrated Traditional Chinese and Western Medicine (review batch number: 2019042).

\section{QSHWC}

The main ingredients of QSHWC included Radix Astragali, Radix Pseudostellariae, Semen Persicae, Flos Carthami, Radix Angelicae Sinensis, Radix Paeoniae Rubra, Rhizoma Chuanxiong, Rhizoma Pinelliae, Radix Scrophulariae, Radix Ophiopogonis, and Radix Achyranthes Bidentatae. Each capsule weighted $0.4 \mathrm{~g}$. QSHWC was produced by the Traditional Chinese Medicine Preparation Room of Hebei Cangzhou Hospital of Integrated Traditional Chinese and Western Medicine (Ji Yao Zhun Zi: Z20050798).

\section{Main reagents and instruments}

The reagents used in the present study included antimicrotubule-associated protein 1 light chain 3 (LC3) antibody (Cell Signaling Technology, MA, USA), antiPI3Kp85 antibody (Santa Cruz, CA, USA), anti-p-PI3Kp85 antibody (Santa Cruz), anti-Akt antibody (Santa Cruz), anti-p-Akt antibody (Santa Cruz), anti-mTOR antibody (Santa Cruz), anti-p-mTOR antibody (Santa Cruz), antiglyceraldehyde-3-phosphate dehydrogenase (GAPDH) antibody (Cell Signaling Technology), and an HE staining kit (ZKWB-Bio Biotechnology Co., Ltd., Beijing, China).

The instruments used in the present study included a small animal ultrasound imaging system (Vevo 3100, Visual Sonics, Canada), a microplate reader (Multiskan FC, Thermo, USA), an inverted microscope (NIKON CI-S, Nikon, Japan), a fluorescence microscope (AZ100, Nikon, Japan), an electrophoretic transfer tank (VE186, Tanon, China), a high-throughput electrophoresis tank (VE180 Mini-protean 3 Dodeca, Thermo, USA), and a high-speed refrigerated centrifuge (TGL-21M, Xiangyi, China).

\section{Study methods}

\section{Model preparation and grouping intervention}

The 48 rats were randomly divided into six groups of eight. The control group was injected with $1 \mathrm{~mL}$ of normal saline through the tail vein. The THP model group received tail 
vein injection of THP once a week [ $3 \mathrm{mg} / \mathrm{kg}$ each time (6)] for 6 consecutive weeks. The low-dose QSHWC (QSHWC-L) group received intragastric administration of QSHWC at $0.125 \mathrm{~g} /(\mathrm{kg} \cdot \mathrm{d})$ for 6 consecutive weeks in addition to 6 consecutive weeks of tail vein injection with THP (once a week, $3 \mathrm{mg} / \mathrm{kg}$ each time). The moderatedose QSHWC (QSHWC-M) group was given a tail vein injection of THP once a week at $3 \mathrm{mg} / \mathrm{kg}$ for 6 consecutive weeks and intragastric administration of QSHWC at $0.25 \mathrm{~g} /(\mathrm{kg} \cdot \mathrm{d})$ for 6 consecutive weeks. The high-dose QSHWC (QSHWC-H) group received a tail vein injection of THP once a week at $3 \mathrm{mg} / \mathrm{kg}$ for 6 consecutive weeks and intragastric administration of QSHWC at $0.5 \mathrm{~g} /(\mathrm{kg} \cdot \mathrm{d})$ for 6 consecutive weeks. The LY294002 group received a tail vein injection of THP (once a week, $3 \mathrm{mg} / \mathrm{kg}$ each time) for 6 consecutive weeks, intragastric administration of QSHWC [0.25 $\mathrm{g} /(\mathrm{kg} \cdot \mathrm{d})]$ for 6 consecutive weeks, and a subcutaneous injection of LY294002 for 6 consecutive weeks at $1.2 \mathrm{mg} /(\mathrm{kg} \cdot \mathrm{d})(7)$.

\section{Echocardiographic examination of the rats}

One day before the rats were sampled, they were anesthetized by intraperitoneal injection with $10 \%$ chloral hydrate and immobilized on the echocardiography table. The rats were subjected to two-dimensional and M-mode echocardiographic examination using the Vevo 3100 small animal echocardiography imaging system. The indices examined included left ventricular end-diastolic diameter (LVEDD), left ventricular end-systolic diameter (LVESD), left ventricular ejection fraction (LVEF), and left ventricular fractional shortening (LVFS).

\section{The pathomorphology of hematoxylin and eosin (HE)-} stained myocardial tissue

Heart tissues were collected from all rats. The tissues were fixed in paraformaldehyde, dehydrated, cleared, infiltrated and embedded in paraffin, cut into 4- $\mu \mathrm{m}$ sections, and routinely stained with HE. The morphology of the myocardial tissue of various groups of rats was examined under an optical microscope.

\section{Immunofluorescence examination of LC3}

The fixed myocardial tissues were permeabilized with Triton X-100 for $20 \mathrm{~min}$, rinsed with water, and incubated with fluorescent dye solution at $37^{\circ} \mathrm{C}$ for $20 \mathrm{~min}$ in the dark. After blocking, the tissues were incubated with fluorescent anti-LC3 primary antibody $(1: 300)$ at $4{ }^{\circ} \mathrm{C}$ overnight in the dark. After washing with phosphate-buffered saline (PBS), the tissues were incubated with fluorescent secondary antibody in a $37^{\circ} \mathrm{C}$ incubator for $30 \mathrm{~min}$. The tissues were then washed with PBS, incubated with 4',6-diamidino-2-phenylindole (DAPI) at room temperature for $15 \mathrm{~min}$, washed again with PBS, and mounted in water. The expression of the protein was examined under a fluorescence microscope.

\section{Western blot analysis of LC3II/I, phosphatidylinositol} 3-kinase (PI3K), p-PI3K, protein kinase B (Akt), p-Akt, mammalian target of rapamycin (mTOR), and p-mTOR Cryopreserved myocardial tissues were ground in liquid nitrogen, and total tissue proteins were extracted using radioimmunoprecipitation assay (RIPA) buffer. Equal amounts of proteins were separated by sodium dodecyl sulfate-polyacrylamide gel electrophoresis and then transferred to membranes. After blocking with 5\% skimmed milk, the membranes were incubated with various antibodies at $4{ }^{\circ} \mathrm{C}$ overnight, including anti-LC3 antibody $(1: 10,000$ dilution), anti-PI3K antibody (1:500 dilution), anti-p-PI3K antibody (1:500 dilution), anti-Akt antibody (1:500 dilution), anti-p-Akt antibody (1:500 dilution), anti-mTOR antibody (1:500 dilution), and anti-p-mTOR antibody (1:500 dilution). Next, the membranes were incubated with secondary antibodies (1:10,000 dilution) at room temperature for $40 \mathrm{~min}$, which was followed by the chemiluminescence reaction with electrochemiluminescence substrate, development, and imaging. The images were subjected to grayscale analysis with the Gel Image System ver.4.00 software.

\section{Statistical processing}

Statistical analysis was conducted in SPSS 22.0 software. Measurement data are expressed as mean \pm standard deviation. Comparison between multiple groups was performed using one-way analysis of variance (ANOVA), while pairwise comparison between the means was carried out using the least significant difference (LSD) $t$-test. A P value of less than 0.05 indicated that the difference was statistically significant.

\section{Results}

\section{The effect of QSHWC on rat heart functions}

LVESD was larger $(\mathrm{P}<0.05)$ while LVEF and LVFS were lower $(\mathrm{P}<0.05)$ in the THP group than the control group. LVESD was shorter $(\mathrm{P}<0.05)$ while LVEF and LVFS were larger $(\mathrm{P}<0.05)$ in the QSHWC-L, QSHWC-M, and QSHWC-H groups than the THP group. LVESD was larger $(\mathrm{P}<0.05)$ while LVEF and LVFS were lower $(\mathrm{P}<0.05)$ 


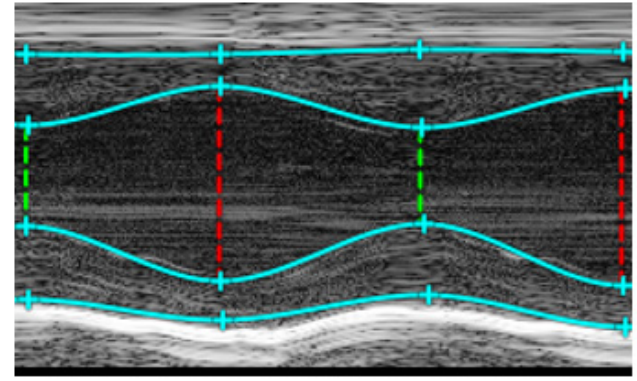

Control

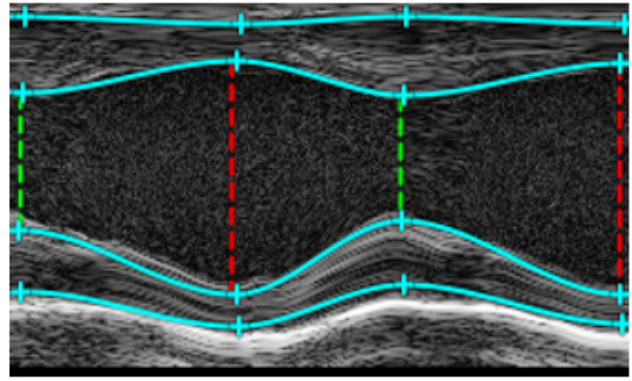

QSHWC-L

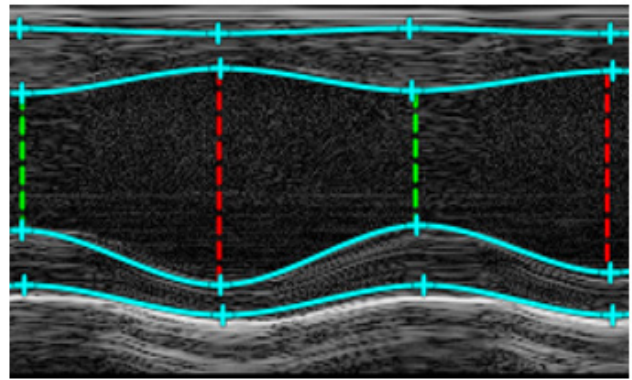

QSHWC-H

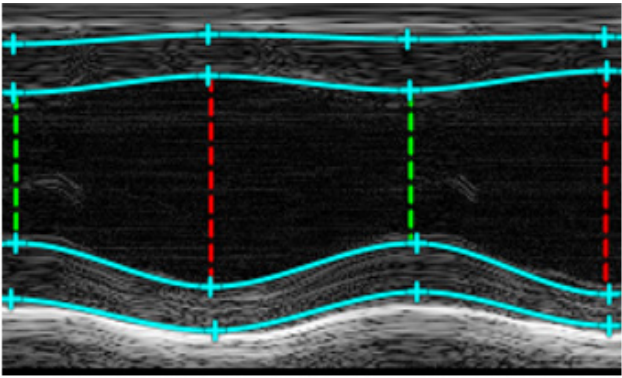

THP

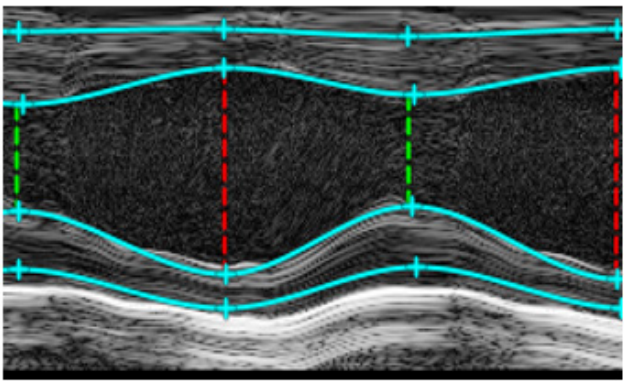

QSHWC-M

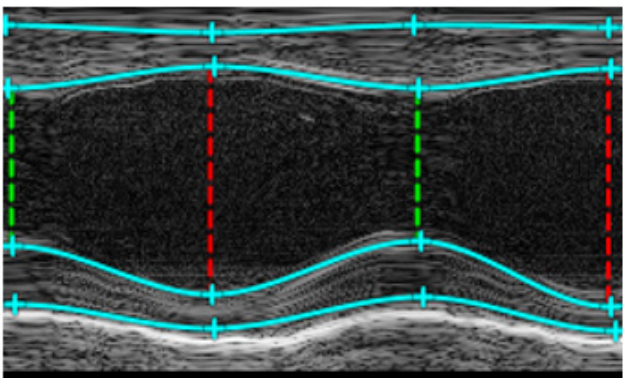

LY294002

Figure 1 Echocardiographic images of all six groups of rats. QSHWC, Qishen Huanwu capsule; THP, pirarubicin.

in the LY294002 group than the QSHWC-M group. The results are shown in Figure 1 and Table 1.

\section{Effect of QSHWC on the morphology of rat myocardial tissue}

Optical microscopy showed that the myocardial fibers of the control group were uniform in thickness and regular in arrangement. The transverse striation of myocardial fibers was clearly visible (Figure 2). In the THP group and the LY294002 group, the thickness of myocardial fibers became uneven, and the transverse striation of the myocardial fibers disappeared. Most of the myocardial fibers appeared hypertrophic. Moreover, the cytoplasmic staining varied in intensity. The nuclei became pyknotic or deformed or disappeared, and myocardial interstitial edema was prominent.
The structure of cardiomyocytes appeared more normal in the QSHWC-L, QSHWC-M, and QSHWC-H groups than with the THP group. In addition, the arrangement of myocardial fibers was more regular, interstitial edema was alleviated, and cell boundaries were clearer in the QSHWC-L, QSHWC-M, and QSHWC-H groups.

\section{Distribution of LC3 in myocardial tissue}

The immunofluorescence results (Figure 3) showed that green fluorescence/LC3 protein expression was higher in the THP group than in the control group. Compared with the THP group, the QSHWC-L, QSHWC-M, and QSHWC-H groups showed less green fluorescence, indicating that they expressed less LC3 protein. Compared 
Table 1 Echocardiographic results of various groups of rats

\begin{tabular}{|c|c|c|c|c|c|}
\hline Group & $\mathrm{n}$ & LVEDD (mm) & LVESD (mm) & LVEF\% & LVFS\% \\
\hline THP & 7 & $8.50 \pm 0.39$ & $5.45 \pm 0.43^{*}$ & $63.28 \pm 4.96^{\star}$ & $35.92 \pm 3.77^{\star}$ \\
\hline QSHWC-L & 8 & $8.18 \pm 0.71$ & $4.80 \pm 0.73^{\#}$ & $70.28 \pm 5.44^{\#}$ & $41.56 \pm 4.56^{\#}$ \\
\hline QSHWC-M & 8 & $7.91 \pm 0.65$ & $4.58 \pm 0.95^{\#}$ & $71.01 \pm 9.87^{\#}$ & $42.49 \pm 8.31^{\#}$ \\
\hline LY294002 & 7 & $8.12 \pm 0.82$ & $5.11 \pm 0.59^{\&}$ & $64.68 \pm 6.34^{\&}$ & $36.94 \pm 5.08^{\&}$ \\
\hline
\end{tabular}

${ }^{*} \mathrm{P}<0.05$ compared with the control group; ${ }^{\mathrm{P}}<0.05$ compared with the THP group; ${ }^{\circledR} \mathrm{P}<0.05$ compared with the QSHWC-M group. LEVDD, left ventricular end-diastolic diameter; LVESD, left ventricular end-systolic diameter; LVEF, left ventricular ejection fraction; LVFS, left ventricular fractional shortening; QSHWC, Qishen Huanwu capsule; THP, pirarubicin.

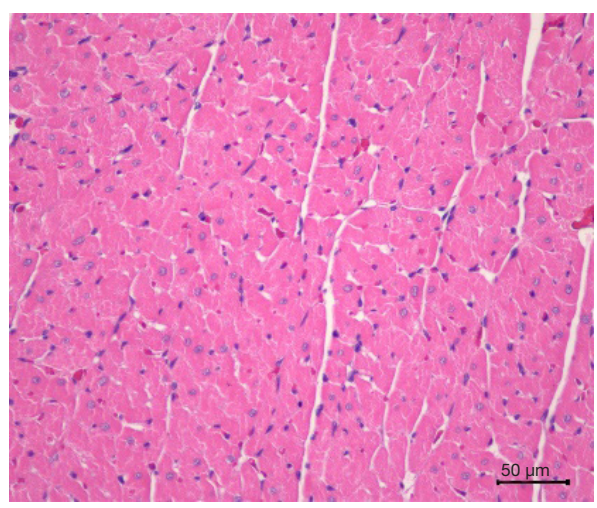

Control

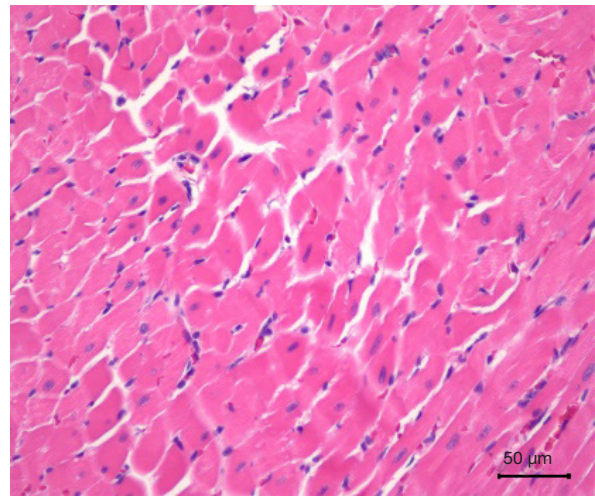

QSHWC-M

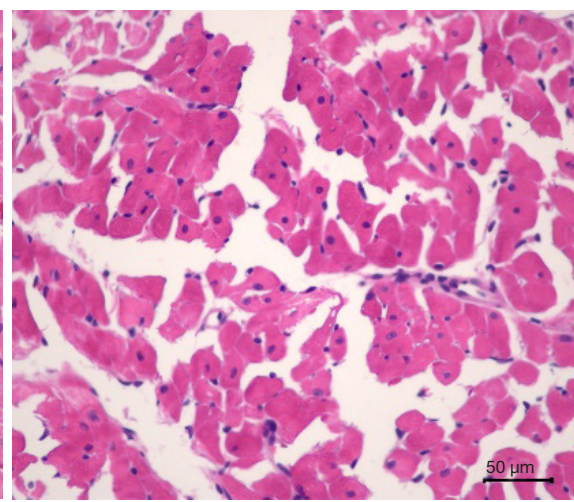

THP

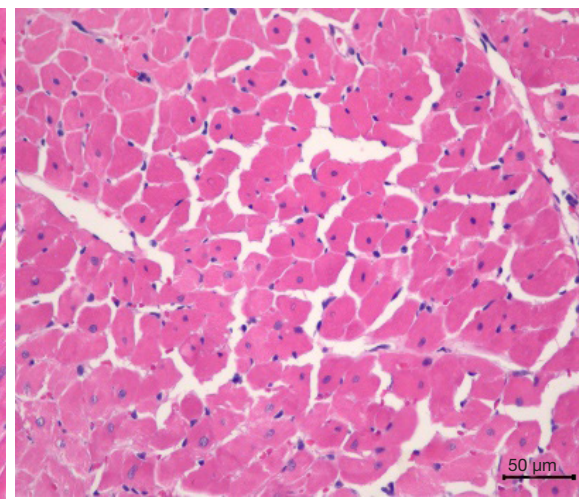

QSHWC-H

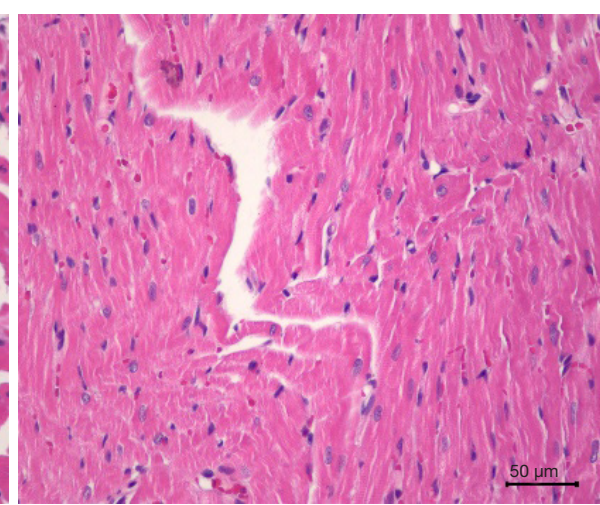

QSHWC-L

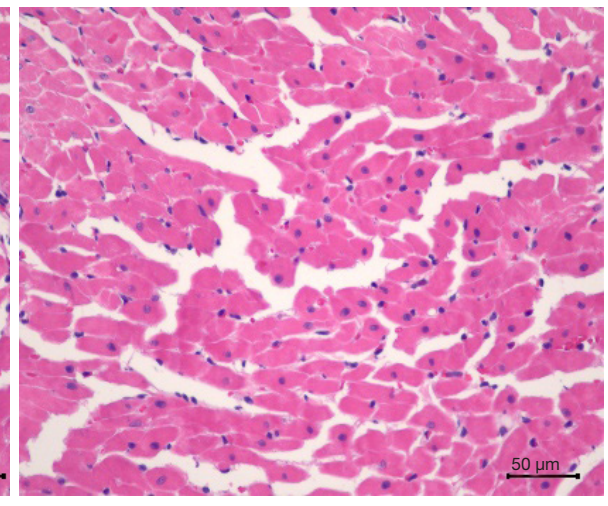

LY294002

Figure 2 HE staining of the myocardial tissues of various groups of rats (×200). QSHWC, Qishen Huanwu capsule; THP, pirarubicin.

with that in the QSHWC-M group, the green fluorescence/ LC3 protein expression was higher in the LY294002 group.

\section{LC3-II/LC3-I ratio in myocardial tissue}

The THP group had a higher ratio of LC3-II/LC3-I than the control $(\mathrm{P}<0.05)$. Compared with THP, treatment with QSHWC reduced the LC3-II/LC3-I ratio to varying degrees. There was a statistically significant difference in LC3-II/LC3-I ratio between the QSHWC-M group and the THP group $(\mathrm{P}<0.05)$. The ratio of LC3-II/LC3-I was significantly higher in the LY294002 group than the 
Control
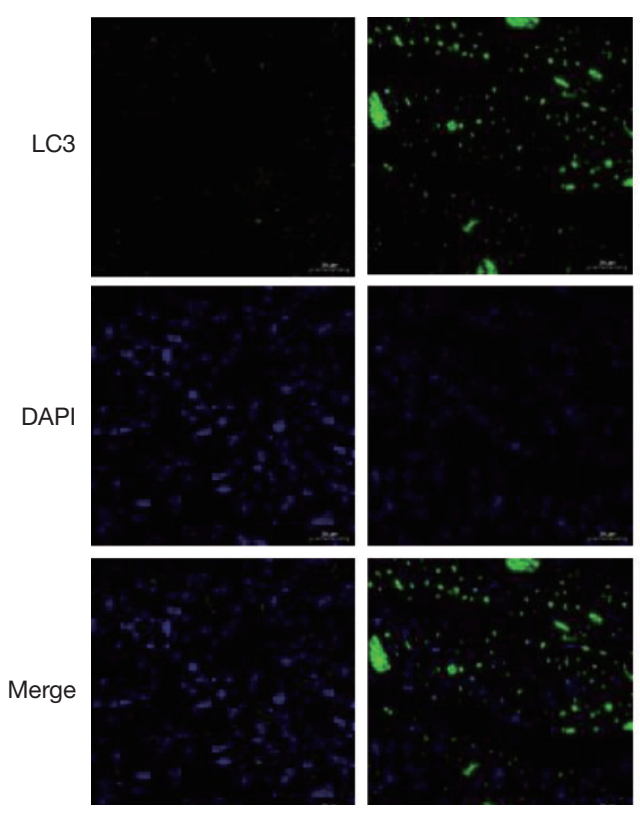

QSHWC-L
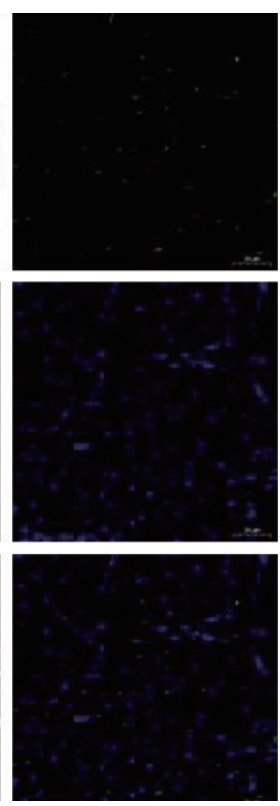

QSHWC-M
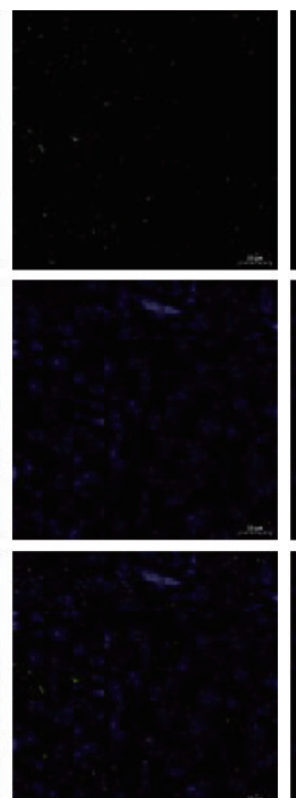

QSHWC-H
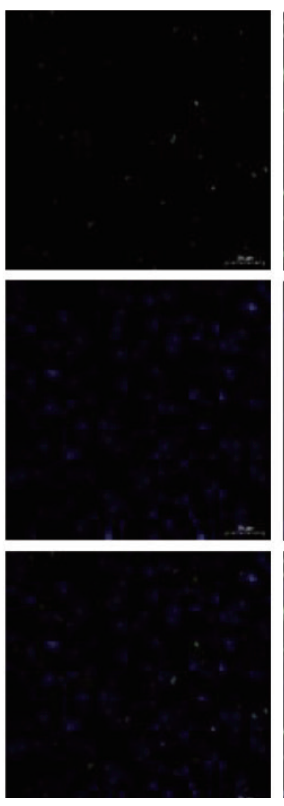

LY294002
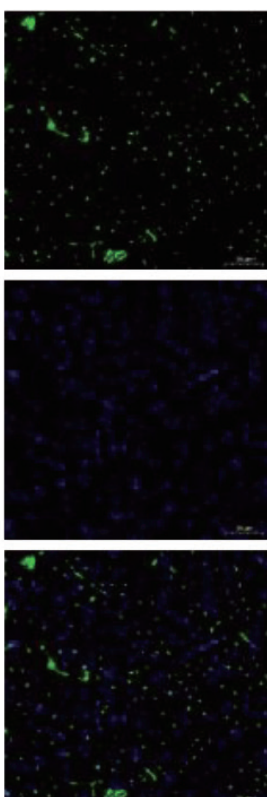

Figure 3 Immunofluorescence staining of LC3 in the myocardial tissues of each group of rats. THP, pirarubicin; QSHWC, Qishen Huanwu capsule; LC3, light chain 3; DAPI, 4',6-diamidino-2-phenylindole.
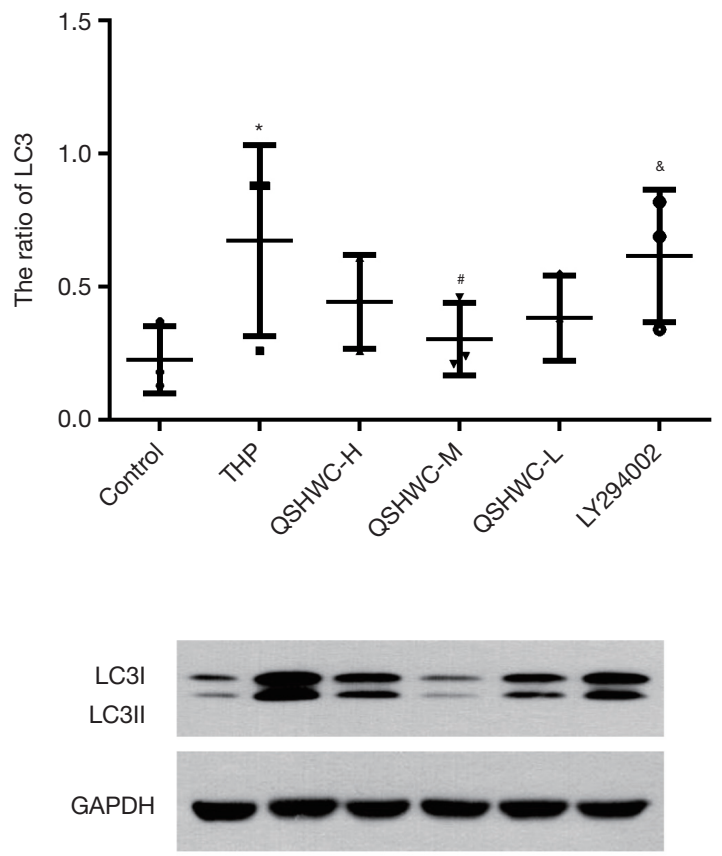

Figure 4 Expression of LC3-II and LC3-I proteins in the myocardial tissues of each group of rats. ${ }^{*} \mathrm{P}<0.05$ compared with the control group; ${ }^{\#} \mathrm{P}<0.05$ compared with the THP group; ${ }^{8} \mathrm{P}<0.05$ compared with the QSHWC-M group. LC3, light chain 3; GAPDH, glyceraldehyde-3-phosphate dehydrogenase; QSHWC, Qishen Huanwu capsule; THP, pirarubicin.
QSHWC-M group $(\mathrm{P}<0.05)$. The results are shown in Figure 4.

Expression status of PI3K, p-PI3K, Akt, p-Akt, mTOR, and $p$-mTOR in myocardial tissue

p-PI3K, p-Akt, and p-mTOR were significantly lower in the THP group than the control group $(\mathrm{P}<0.05)$. p-PI3K, p-Akt, and p-mTOR were significantly higher in the QSHWC-M group than the THP group $(\mathrm{P}<0.05)$, indicating that QSHWC activated the PI3K/Akt/mTOR pathway. LY294002 significantly reversed the above effects $(\mathrm{P}<0.05)$. There was no significant difference in the expression level of PI3K, Akt, or mTOR between the six groups. The results are shown in Figure 5.

\section{Discussion}

Cardiotoxicity is one of the most serious side effects of anthracyclines such as THP. The exact mechanism by which anthracyclines induce cardiotoxicity remains unclear. The molecular mechanisms of anthracyclines involve multiple aspects, including lipid peroxidation, accumulation of reactive oxygen species, DNA damage, and mitochondrial dysfunction (8). Autophagy dysfunction plays 

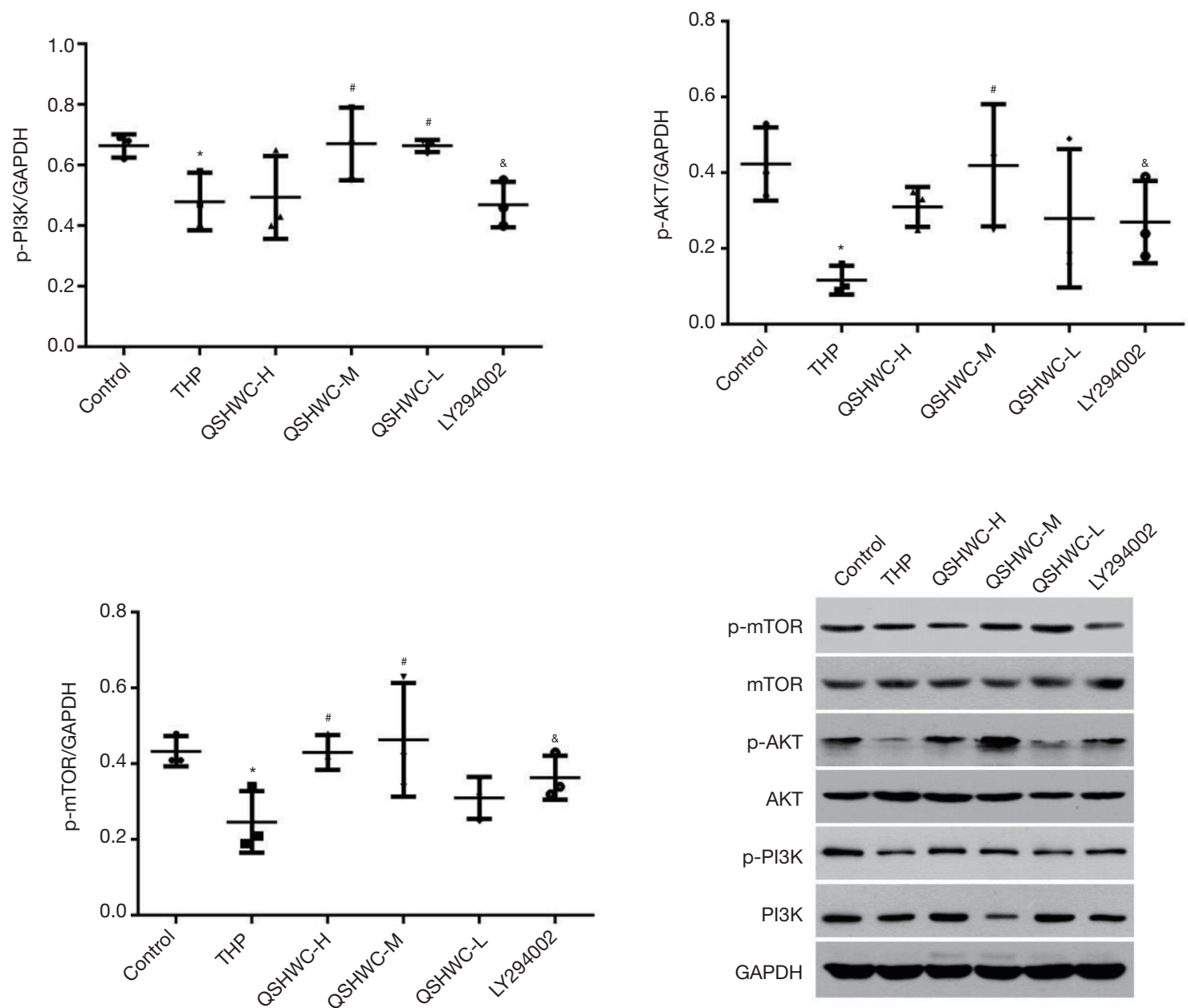

Figure 5 Expression status of PI3K, p-PI3K, AKT, p-AKT, mTOR, and p-mTOR in the myocardial tissue of each group of rats. ${ }^{*} \mathrm{P}<0.05$ compared with the control group; ${ }^{\#} \mathrm{P}<0.05$ compared with the THP group; ${ }^{\circledR} \mathrm{P}<0.05$ compared with the QSHWC-M group. PI3K, phosphatidylinositol 3-kinase; p-PI3K, phosphorylated-PI3K; AKT, protein kinase B; mTOR, mammalian target of rapamycin; THP, pirarubicin; QSHWC, Qishen Huanwu capsule.

an important role in the cardiotoxicity of anthracyclines (9). This study intended to explore the potential mechanism by which QSHWC reduced THP-induced cardiotoxicity by examining autophagy.

The exact role of autophagy in the cardiotoxicity of anthracyclines is not clear. Anthracyclines upregulate autophagy-related gene 5 (Atg-5) and other autophagyrelated genes, upregulate the autophagy biomarker protein LC3-II, and promote the formation of autophagosomes in cardiomyocytes, resulting in cardiomyocyte death. These findings indicate that the upregulation of autophagy level plays a detrimental role in anthracycline-induced cardiotoxicity (10). However, the use of anthracyclines leads to the downregulation of LC3-II protein expression level (11). In the present study, the results of immunofluorescence staining showed that the LC3 signal was significantly higher in the THP group than in the control group, and western blot showed that the expression level of LC3-II protein was significantly higher in the THP group than in the control group. Such results indicate that autophagy was overactivated in cardiomyocytes by THP. In the THP group, LVEF was significantly lower than that in the control group, and the morphology of myocardial fibers was significantly altered, suggesting that the excessive activation of autophagy was involved in the THP-induced myocardial damage. 
QSHWC is derived from a classic prescription of traditional Chinese medicine, Buyang Huanwu decoction. QSHWC alleviates the pituitrin-induced cardiomyocyte necrosis and myocardial interstitial fibrosis in rats. To explore whether QSHWC improves myocardial damage caused by THP, we subjected various groups of rats to echocardiographic examination and HE staining of myocardial tissue. The results showed that the LVESD, LVEF, and LVFS were all better in the QSHWC-L, QSHWC-M, and QSHWC-L groups compared with the THP group. In addition, in the QSHWC-L, QSHWC-M, and QSHWC-L groups, the structure of cardiomyocytes was relatively normal, the arrangement of myocardial fibers was more regular, and there was less interstitial edema, suggesting that QSHWC alleviated the myocardial damage caused by THP.

LC3 has two forms: LC3-I and LC3-II. When intracellular autophagy occurs, LC3-I protein will be converted into LC3-II. Therefore, the level of LC3-II reflects, to some extent, the degree of autophagy (12). The THP rats had an elevated number of green-fluorescent LC3 puncta and ratio of LC3-II/LC3-I, suggesting that their cardiomyocytes had a large number of autophagosomes and an increase in autophagy. The QSHWC-L, QSHWC-M, and QSHWC-L groups had fewer green fluorescent spots and a lower LC3-II/LC3-I ratio than the THP group, indicating that QSHWC reduced the formation of autophagosomes and that QSHWC might exert its myocardial-protective effect through inhibition of the autophagy of cardiomyocytes.

The regulatory process of autophagy is rather complicated. The key players in autophagy initiation are the mTOR-related targets, which are regulated by various signaling pathways, such as the PI3K/Akt pathway (13). The PI3K/Akt pathway is the main regulator of cell growth and survival. It plays an important role in the survival of cardiomyocytes under many circumstances. Activation of the PI3K/Akt pathway phosphorylates mTOR, thereby inhibiting autophagy. The PI3K/Akt pathway is one of the most important upstream pathways of mTOR (14). Buyang Huanwu decoction, the basic prescription of QSHWC, upregulates the PI3K/Akt pathway (15). In our previous study, network pharmacology was used to predict the mechanism by which QSHWC reduced the toxicity of anthracyclines. Kyoto Encyclopedia of Genes and Genomes (KEGG) pathway enrichment analysis revealed that the main signal transduction pathway enriched was the PI3K/ Akt/mTOR pathway.
To explore the mechanism by which QSHWC reduced the THP-induced excessive activation of cardiomyocyte autophagy, we performed western blots for p-mTOR, mTOR, p-Akt, Akt, p-PI3K, and PI3K in the myocardium. p-mTOR, p-Akt, and p-PI3K were significantly lower in the THP group than the control group. QSHWC upregulated p-mTOR, p-Akt, and p-PI3K, while the PI3K inhibitor LY294002 significantly reversed the above effects of QSHWC. These results indicate that QSHWC inhibited excessive autophagy by activating the PI3K/Akt/mTOR pathway.

In summary, QSHWC activates the PI3K/Akt/ mTOR pathway to inhibit the excessive autophagy of cardiomyocytes caused by THP, thereby exerting a protective effect on the myocardium, reducing THPinduced myocardial damage, and improving cardiac function.

\section{Acknowledgments}

Funding: None.

\section{Footnote}

Reporting Checklist: The authors have completed the ARRIVE reporting checklist. Available at http://dx.doi. org/10.21037/apm-20-1746

Data Sharing Statement: Available at http://dx.doi. org/10.21037/apm-20-1746

Conflicts of Interest: All authors have completed the ICMJE uniform disclosure form (available at http://dx.doi. org/10.21037/apm-20-1746). The authors have no conflicts of interest to declare.

Ethical Statement: The authors are accountable for all aspects of the work in ensuring that questions related to the accuracy or integrity of any part of the work are appropriately investigated and resolved. All the procedures were carried out according to the Guide for the Care and Use of Laboratory Animals published by the U.S. National Institutes of Health and were reviewed and approved by the Ethics Committee of Cangzhou Hospital of Integrated Traditional Chinese and Western Medicine (review batch number: 2019042).

Open Access Statement: This is an Open Access article 
distributed in accordance with the Creative Commons Attribution-NonCommercial-NoDerivs 4.0 International License (CC BY-NC-ND 4.0), which permits the noncommercial replication and distribution of the article with the strict proviso that no changes or edits are made and the original work is properly cited (including links to both the formal publication through the relevant DOI and the license). See: https://creativecommons.org/licenses/by-nc-nd/4.0/.

\section{References}

1. Valcovici M, Andrica F, Serban C, et al. Cardiotoxicity of anthracycline therapy: Current perspectives. Arch Med Sci 2016;12:428-35.

2. Zhang Y, Ma XY, Zhang T,et al.Protective Effects of Apocynum venetum Against Pirarubicin-Induced Cardiotoxicity. Am J Chin Med 2019;47:1075-97.

3. Koleini N, Kardami E. Autophagy and mitophagy in the context of doxorubicin-induced cardiotoxicity. Oncotarget 2017;8:46663-80.

4. Chen X, Peng X, Luo Y, et al. Quercetin protects cardiomyocytes against doxorubicin-induced toxicity by suppressing oxidative stress and improving mitochondrial function via 14-3-3 $\gamma$. Toxicol Mech Methods 2019;29:344-54.

5. Xiao J, Sun GB, Sun B, et al. Kaempferol protects against doxorubicin-induced cardiotoxicity in vivo and in vitro. Toxicology 2012;292:53-62.

6. Li Q, Qin M, Li T, et al. Rutin protects against pirarubicin-induced cardiotoxicity by adjusting microRNA-125b-1-3p-mediated JunD signaling pathway. Mol Cell Biochem 2020;466:139-48.

7. Li X, Hu X, Wang J, et al. Inhibition of autophagy via activation of PI3K/Akt/mTOR pathway contributes to

Cite this article as: Wang F, Wang L, Wang Z, Liu H. Qishen Huanwu capsule reduces pirarubicin-induced cardiotoxicity in rats by activating the PI $3 \mathrm{~K} / \mathrm{Akt} / \mathrm{m}$ TOR pathway. Ann Palliat Med 2020;9(5):3453-3461. doi: 10.21037/apm-20-1746 the protection of hesperidin against myocardial ischemia/ reperfusion injury. Int J Mol Med 2018;42:1917-24.

8. Dos Santos Arruda F, Tomé FD, Miguel MP, et al. Doxorubicin-induced Cardiotoxicity and Cardioprotective Agents: Classic and New Players in the Game. Curr Pharm Des 2019;25:109-18.

9. Lee EY, Yan AT. Looking beyond cancer for cabozantinibinduced cardiotoxicity: evidence of absence or absence of evidence? Ann Transl Med 2019;7:S121.

10. Sun A, Cheng Y, Zhang Y, et al. Aldehyde dehydrogenase 2 ameliorates doxorubicin-induced myocardial dysfunction through detoxification of 4-HNE and suppression of autophagy. J Mol Cell Cardiol 2014;71:92-104.

11. Dutta D, Xu J, Dirain ML, et al. Calorie restriction combined with resveratrol induces autophagy and protects 26-month-old rat hearts from doxorubicin-induced toxicity. Free Radic Biol Med 2014;74:252-62.

12. Klionsky DJ, Abdelmohsen K, Abe A, et al. Guidelines for the use and interpretation of assays for monitoring autophagy (3rd edition). Autophagy 2016;12:1-222.

13. Varshney P, Saini N. PI3K/AKT/mTOR activation and autophagy inhibition plays a key role in increased cholesterol during IL-17A mediated inflammatory response in psoriasis. Biochim Biophys Acta Mol Basis Dis 2018;1864:1795-803.

14. Gao Y, Zhang Y, Fan Y. Eupafolin ameliorates lipopolysaccharide-induced cardiomyocyte autophagy via $\mathrm{PI} 3 \mathrm{~K} / \mathrm{AKT} / \mathrm{mTOR}$ signaling pathway. Iran J Basic Med Sci 2019;22:1340-6.

15. Yin ZF, Wei YL, Wang $X$, et al. Buyang Huanwu Tang inhibits cellular epithelial-to-mesenchymal transition by inhibiting TGF- $\beta 1$ activation of PI3K/Akt signaling pathway in pulmonary fibrosis model in vitro. BMC Complement Med Ther 2020;20:13. 